\title{
Human Fall Detection Using Video Surveillance
}

\author{
Kavya G, Sunil Kumar C T, Dhanush C and Kruthika J \\ Research Scholar, Visvesvaraya Technological University, Karnataka, India \\ DOI: https://doi.org/10.34293/acsjse.v1i1.1
}

\begin{abstract}
Fall is one of the biggest challenge in elderly people, pregnant and small children's, who stays alone in home. Sometimes this fall leads to severe injuries and even to death. Detecting the fall is very much important for elderly people. Convolutional Neural Network (CNN) is an deep learning algorithm used for image processing. In this paper, we present a video-based fall detection using CNN, this CNN will perform background subtraction and captures only foreground objects to detect the human movements and detect if fall happens. Firstly, camera will be capturing all the movements of the person. Our proposed model will detect the fall and finally an alarm is raised and email is sent to a given particular caretaker and family member. Our experimental results show the best performance of the proposed model.
\end{abstract}

Keywords: Fall detection, video-based, Convolutional Neural Network, Elderly people, Smart home.

\section{INTRODUCTION}

Health condition of the elderly people will not be constant all the time. Since they might be suffering from some of the common diseases, the accidental fall may lead to severe injury or sometimes death. There are already sensor based devices such as accelerometer, watch to call when they need help. But, sometimes elder people will forget to wear this sensor devices or can't able to press the button by becoming unconscious after the sudden fall. Elder people may even get heart attack from this sudden fall shock.

According to the survey there are more than 2.8 billion aged people are treated as emergency due to fall injuries. Some of the common chronic diseases reported for aged people includes heart disease, diabetes, depression, stroke, cancer and many more. Due to the above mentioned chronic diseases some of the people may loss their balance, fainting or loss of consciousness, feeling of dizziness which may lead to fall. Although ambience device are installed on elder people to detect their movements, the main defect in this is that, it even detects the non object noise signals, and gives false alarm. Wearable sensors will use accelerometer to detect the fall, the main drawback here is sometimes the elder people will forget to wear the sensors, if they fall without wearing this sensors then nobody will get to know about this fall. Computer vision-based will use one or more cameras placed to detect the fall. Here the cameras are placed so that it will be monitoring the activities of the elder people continuously, the main challenge in this is to require an high processing power for real-time videos and sometimes the daily activities like sleeping, bending down this are all the similar like fall, this method should not consider this type of activities as fall.

To overcome this drawback, in this paper, we used video-based fall detection using Convolution Neural Network (CNN). This CNN eliminates all the background objects and concentrates only on foreground objects. $\mathrm{CNN}$ is applied on each frames of the video to learn the human activities and body features for fall detection. 
This respective paper is organised as follows: section II is the related work with respect to fall detection. Section III is of Methodology presented in detail. Section IV explains about the performance evolution. Section $\mathrm{V}$ gives an experimental results and discussions. Conclusion is provided in section VI. And finally the References that we have used is presented in section VII.

\section{RELATED WORK}

There are basically several studies which tends to explore how falls occur and how to detect when fall takes place since there is a need for emergency treatment whenever these types of unexpected falls occur. Sometimes falls may be serious and can cause death. So proper measures and treatments should be provided. To make it happen we should monitor the movements of human and raise alarm. This method was proposed by several papers those are listed below.

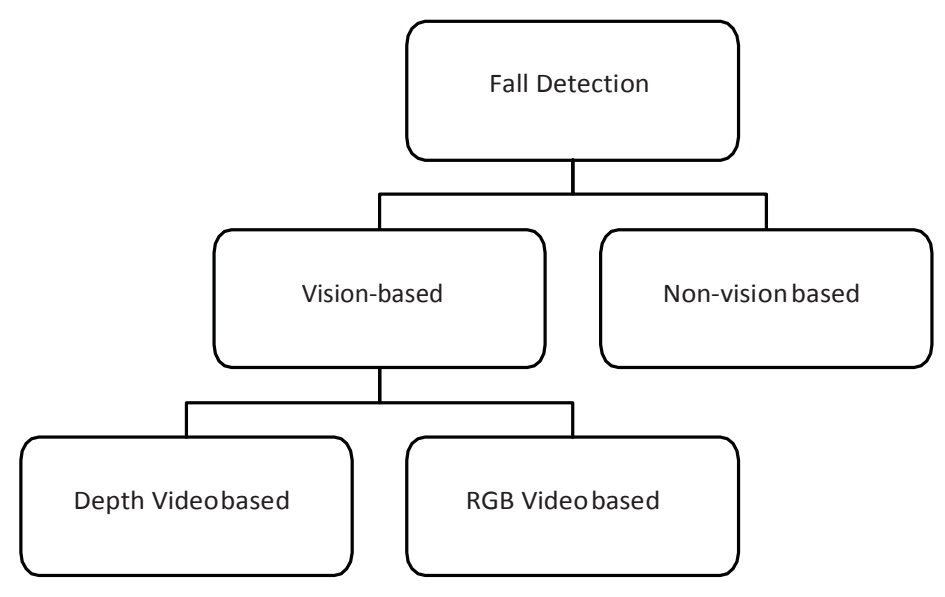

Figure 1: Types of recognising the fall detection

In [1] paper the human fall is detected using relevant pixel-based features. This paper provides the combined GLR detection scheme with respect to the SVM classification. It distinguished between daily activities(false-fall) and falls(true-falls)than other approaches. This approach lacks in depth imagery and preserved privacy for people.

Human fall is detected using visual-surveillance in [2]. OpenCV Library and Improved GMM is used in this approach and performance is evaluated on 21 videos dataset. The problem is human fall can't be detected close to camera and parallel to camera.

The proposed work is based on two techniques, an Ellipse approximation and Motion History Image (MHI).It proved that when compared to individual techniques, combined techniques gives much better accuracy and also efficiency in detecting a human fall.

In paper [4] a three-stage camera-based model is proposed in terms of good accuracy and the low processing time, to reduce the chances of human fall. They are working to improve human object segmentation algorithm and apply three-dimensional features.

Paper [5] is of a novel based on 2D video-based fall detection pipeline with respect to human pose estimation. The researchers are still working and are conducting more experiments of human fall detection in dark environment i.e., at night. 
The paper [6] presents the use of Convolutional Neural Networks(CNN) for automatic fall detection in video surveillance environment with high accuracy. Many experiments are conducted to analyse a drawback in this approach that degrades the performance when backgrounds and foregrounds are changed.

In many of the cases the algorithms are used to get the desired outcome. These algorithms are currently adopted by many of the systems and are used previously to get required output.

\section{MeTHODOLOGY}

The goal of this proposed method is to analyse the movements of human and detect the fall if occurs. This has been mainly designed for indoor environment wherein falls will be detected and respective notification will be sent via mail or registered number. There is certain method we have followed in order to make this success. The flow chart is as shown below.

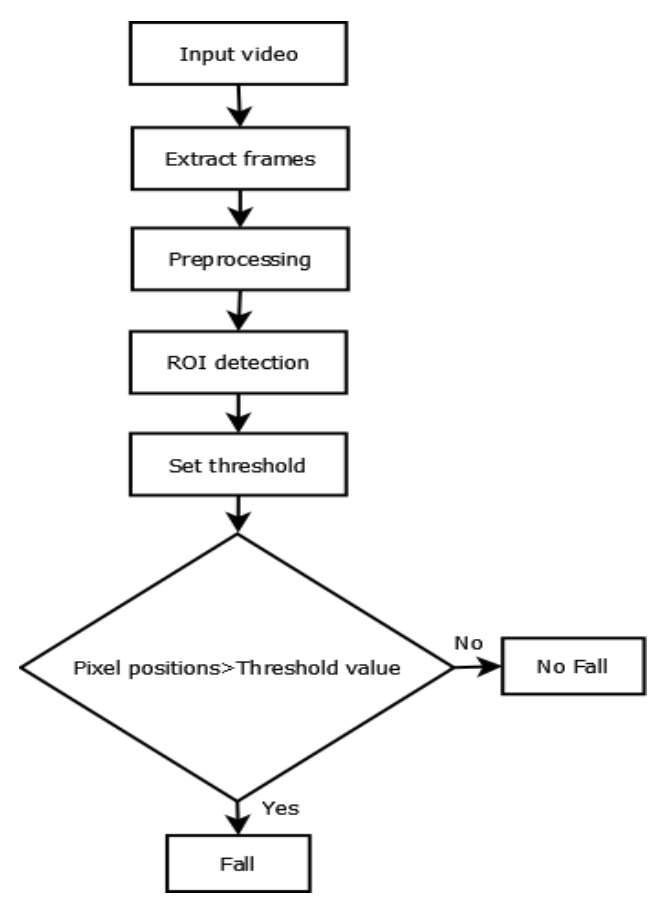

Figure 2: Flow Chart of the proposed method

As we can see above there are certain steps that includes in detecting fall that has been occurred. These steps includes:

Step 1: INPUT VIDEO - In this step respective videos are given as input and is made to detect the fall. These videos may be given either from the videos that are recorded or directly from the camera that we have installed.

Step 2: EXTRACT FRAMES - Every time videos can't be paused and seen for fall. Therefore we are going to extract frames from the given video. Here, Key Frame Extraction is used wherein 10 frames are extracted from video in 60 seconds, frames are extracted continuously and is sent to detect fall in further steps. 
Step 3: PREPROCESSING - It is one of the major step that has been carried out using Open CV. In pre-processing we use background subtraction algorithm to overcome from the unwanted background those are in video frames.

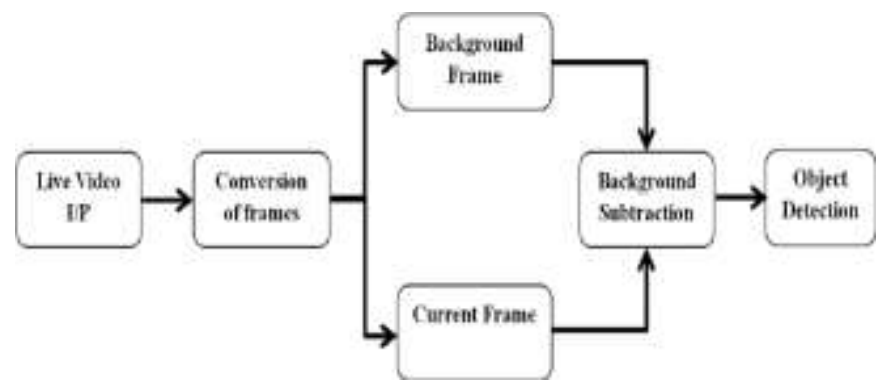

Figure 3: Working of background subtraction

The above figure clearly shows how pre-processing works. Here, basically the videos are given as input and these videos are converted to frames. Immediately, after this conversion background subtraction algorithm works and removes unwanted background. After removing background it detects human.

Step 4: ROI DETECTION - As explained above Background Subtraction is used to detect Region Of Interest (ROI). There are two main steps that takes place in ROI. The initial step is of colour transformation, basically in which the RGB value of frames extracted, here every pixel of frames is transformed to gray value. Next step is about shape matching of gray images to find the possible shape of human.

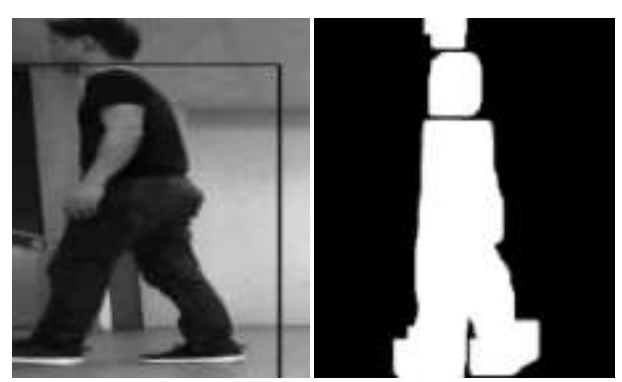

(a)

(b)

Figure 4: (a) the figure which describes actual image after recording i.e., the raw image (b) the figure obtained after ROI detection

Step 5: SET THRESHOLD - In order to detect the fall certain threshold values are set and, the height and width of the person is calculated. Firstly, height of a person is calculated by taking the values of the top most pixel and the value of the bottom most pixel of the image in which ROI is detected. Then the datasets are observed and required heuristics is applied accordingly. In case there is a fall almost all the pixel which belongs to that person starts to move in downward direction and hence the fall is detected.

Step 6: In this step the pixel position values and the threshold values are taken into consideration. When the pixel values are greater than the threshold values the fall will be confirmed if not there will be no fall. 
Step 7: The above same steps will be carried out for all the frames that has been extracted.

\section{Alarm Rising}

After fall has been successfully detected then notification will be sent via email using Mailgun technology and SMS will be sent to respective registered numbers via twilio library and call will be triggered as alarm for registered number. So that the emergency can be treated and this leads to the successful completion of this proposed system.

\section{Algorithm Used}

The algorithm used to approach this technology is as shown in Fig 6. In this algorithm certain files such as video, time are imported in order to understand easily. These files contain the algorithms according to the input video processing and providing desired result.

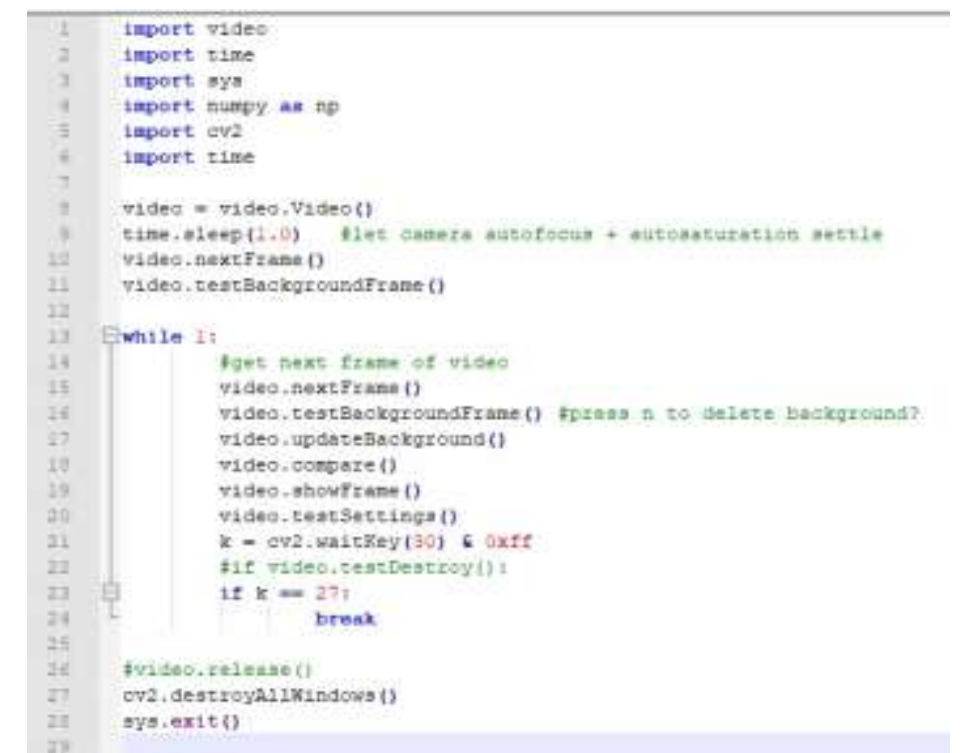

Figure 5: Algorithm Used.

\section{PERFORMANCE EVOLUTION}

Every system performs its own respective tasks. Similarly, in this proposed method the video is divided into frameworks to recognise the fall of human. Technically, there are several phases to recognize the incident of human fall over real-time. Firstly, the video which is recorded is given as the input then frames are extracted from these videos and then background subtraction is performed inorder to extract the desired target i.e., shape of human. Here, Background subtraction is mainly used because of its advantages such as it takes less time in processing and provides improved segmentation and will accurately differentiates between the non human and human objects. Further, the height and width ratio of a person is calculated by considering the distances between the mid-centre and top of rectangle inorder to recognize the human fall, incase if the result of height and width ratio is less than calculated threshold value then, fall incident is detected. If human is inactive for few seconds, then the fall has been confirmed and for the same and alarm is triggered as an alert to 
caretakers and family members and mail is sent to them using mailgun API. And even an SMS and call alert is sent by using twilio library.

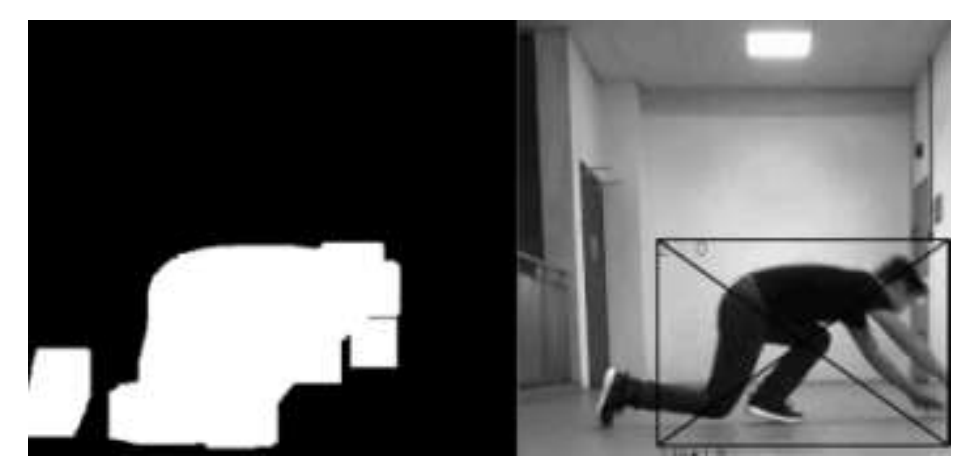

(a)

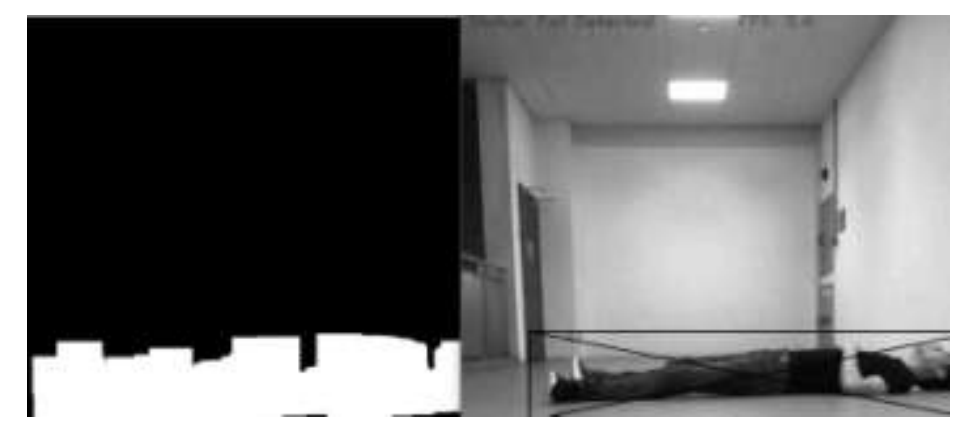

(b)

Figure 6: (a) This fig describes the initial process in which fall occurs; (b) This image is of conformation of fall that has been occurred and further is carried out by alarm triggering

\section{A. Foreground Extraction of Objects}

Here, the Background subtraction algorithm is applied for segmentation of foreground objects and thus the desired target is obtained by detecting the moveable objects. This method will reduce the memory requirements of algorithms, increases its computational efficiency.

\section{B. Background Subtraction}

This techniques allows the image to capture foreground object is extracted for further processing. Foreground detection will separate foreground object from background.

In our proposed model, it provides highly effective performance efficiency. Algorithm used in our model is Convolution Neural Network (CNN), CNN is used for predicting the movement of a human based on sensor data and it performs deep domain expertise and methods from signal processing. It takes information from raw data to fit a machine learning model. An threshold value is set as default, If the value is less than the threshold value then it is detected that human fall has happened.

The score that has been matched is used accordingly to estimate the pose of human body. Here, the Score that has been matched has less deviation in video frames till the changes that suddenly takes place in human body. 
When the match score is changed quickly, then the height and width ratio and the distance between the middle center and top center will be changed and hence leads in predicting the human fall. Basically, the height of human is considered to be 3-4 times more as compared to human width. Moreover, in some of the cases the human may sit or crouch down, even in this type of human position the height will be reduced and the problem is we can't consider these positions as fall. In order to overcome this mistake certain threshold values are set and the application is designed in such a manner that only the fall will be detected no wrong assumptions will be made when the human is sitting or bending.

\section{RESULTS AND DISCUSSIONS}

In proposed system we have used OpenCV Library as a tool and performed on video dataset. The information of 30 daily usual human activities and 21 unusual human activities are obtained from the video sequences. Some of the unusual activities of human such as backwardlsidewaylforward fall may occur due to loss of balance.

When a video is given as input then IOT is shown that a human enters inside the room and shows the chances of falling activity, when human is walking he is presented by green coloured rectangle and if human falls then it is represented by red coloured rectangle. This model preforms background subtraction method and remove all the non-moveable objects and detect the humans. After fall happens, if the human did not stand, after few seconds human fall is deducted, An alarm is triggered and uses mailgun API for sending mails to the respective caretakers and to the family members, even an SMS and call service is provided so that they can get alert even when they are offline. Twilio library is used to send an SMS and call to caretaker and family members.

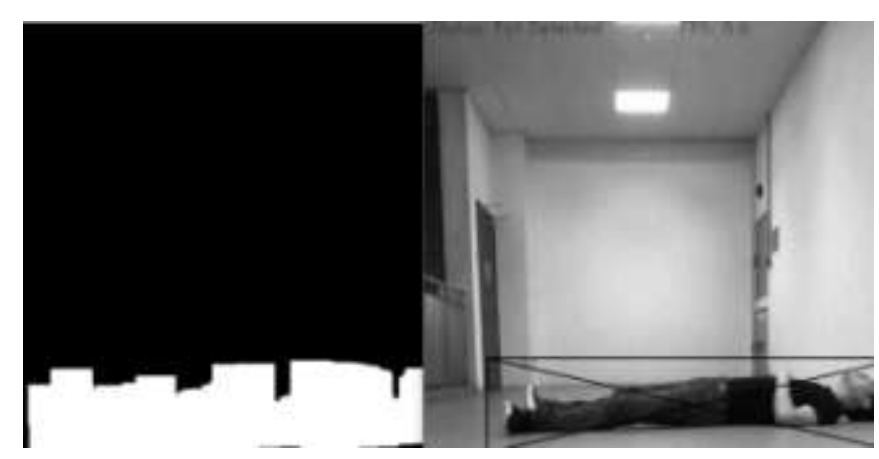

Figure 7: Fall has been occurred and confirmed that the person is not moving

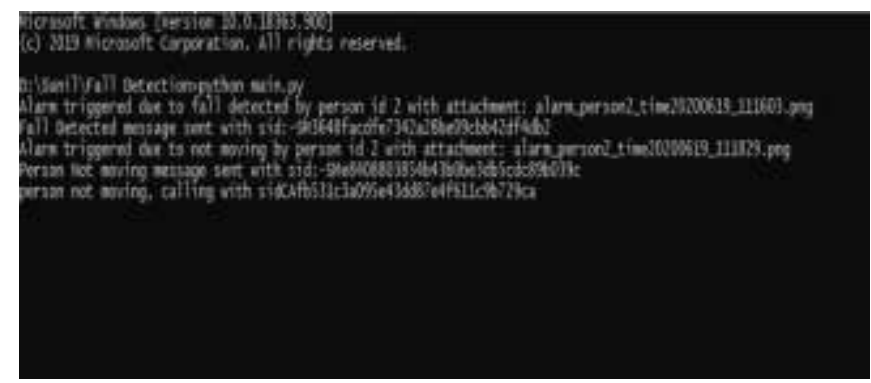

Figure 8: Command prompt that will be executed in the background when fall occurs, this is mainly done to trigger alarm 


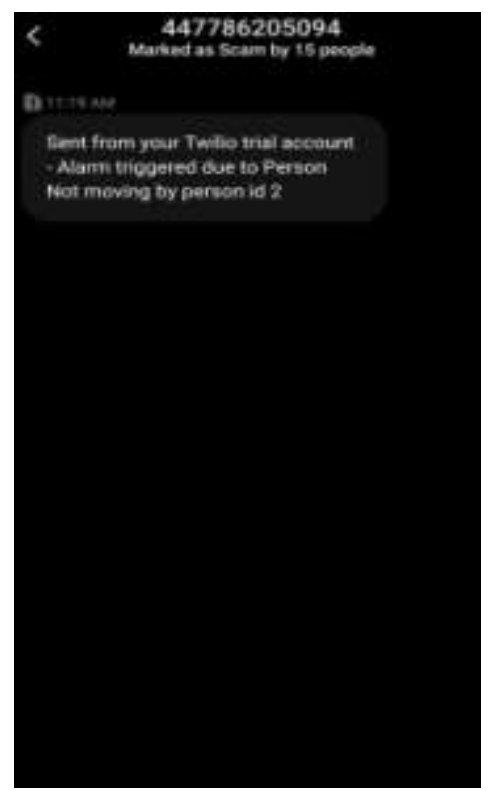

Figure 9: SMS generated to the respective registered number when fall occurs

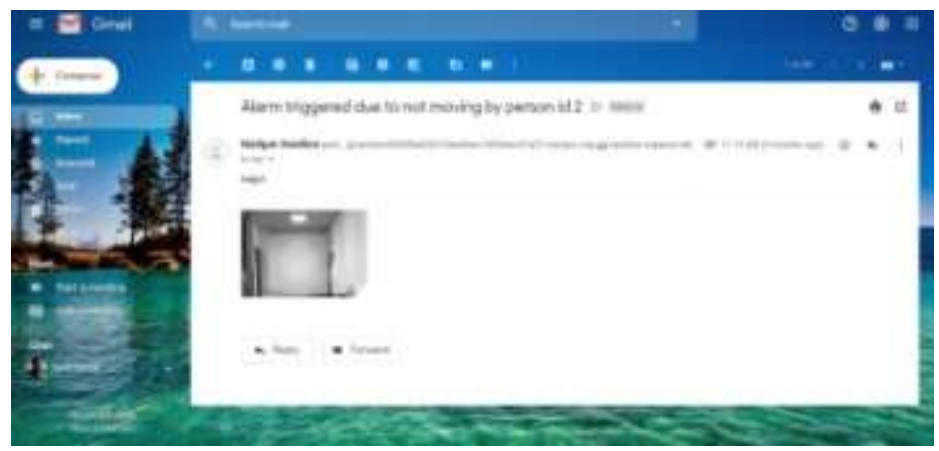

Figure 10: This image is of online service wherein the mail is sent to respective mail id when fall occurs and the person is not moving

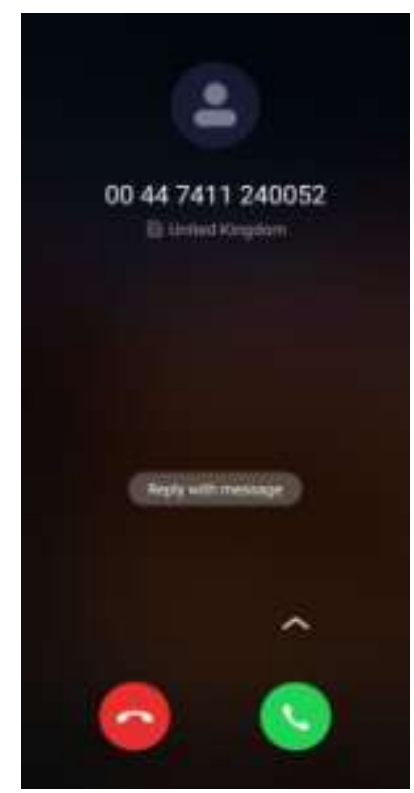

Figure 11: Call triggered as alarm when fall occurs 


\section{CONCLUSION}

This proposed system Methodology of Fall Detection is mainly designed for the elder people who can't always be under someone's care. This category may also involve children whose parents are outside and can also include pregnant womens who can't always do their work especially in that period of time, and also we can't determine what's going to happen for these people when they are in home and unfortunately if something bad happens these people can't contact they may unable to get up. At this type of critical time imagine someone always staying beside you and helps you when something unexpected

Happens. Exactly, this is actually main aim of this project here. Since humans can't stay beside someone permanently we introduce machines to learn about human activities and report whenever a fall occurs so that the machine can automatically raise emergency alarm and can be treated for the same. Here the Region of Interest(ROI) is detected and the threshold value is calculated and this value is compared with the pixel values. Based on this the fall is confirmed. Once, the fall is confirmed the alarm is raised and the notification is sent to the respective person's mail id through mailgun and SMS is sent to the registered number via twilio.

\section{REFERENCES}

[1] United Nations Population Fund (UNFPA) and Help Age International. "Ageing in the Twenty-first Century: A Celebration and a Challenges," New York, 2012.

[2] Falls Among Older Adults: An Overview 2012

[3] Gurley RJ, N Lum, M Sande, and MH Katz. "Persons found in their homes helpless or dead," N Engl J Med., 1996.

[4] Bogdan Kwolek, and Michal Kepski. "Human fall detection on embedded platform using depth maps and wireless accelerometer," Computer Methods and Programs in Biomedicine, 117(3), 2014, pp. 489-501.

[5] X. Ma, H. Wang, B. Xue, M. Zhou, B. Ji, and Y. Li. "Depth-based human fall detection via shape features and improved extreme learning machine," IEEE J. Biomed. Health Informat., 18(6), 2014, pp. 1915-1922.

[6] Takumi Kaneko, and Meifen CAO. "Human Fall Detection Using CHLAC Features with Skeletal Image Sequences," IEEE International Symposium on Robotics and Intelligent Sensors (IRIS2016), 2016.

[7] Hong Thi Khanh Nguyen, Hassoon Fahama, Cecile Belleudy, and Tuan Van Pham. "Low Power Architecture Exploration for Standalone Fall Detection System Based on Computer Vision," UKSim-AMSS 8th European Modelling Symposium, 2014

[8] J. Willems, G. Debard, B. Vanrumste, and T. Goedeme. "A video-based algorithm for elderly fall detection," World Congress on Medical Physics and Biomedical Engineering, Springer Berlin Heidelberg, 2009, pp. 312-315.

[9] Yie-Tarng Chen, Yu-Ching Lin and Wen-Hsien Fang. "A hybrid human fall detection scheme," IEEE 17th International Conference on Image Processing, 2010

[10] Yiwen Su, Daliang Liu, and Yingfeng Wu. "A Multi-sensor Based Pre-impact Fall Detection System With a Hierarchical Classifier," 9th International Congress on 
Image and Signal Processing, BioMedical Engineering and Informatics(CISP-BMEI), 2016, pp.1727-1731.

[11] S.J. Preece, J.Y. Goulermas, L.P.J. Kenney, D. Howard, K. Meijer, and R. Crompton. "Activity identification using body- mounted sensors - a review of classification techniques," Physiological Measurement, 2009.

[12] Laura Montanini, Antonio Del Campo, Davide Perla, Susanna Spinsante, and Ennio Gambi. "A footwear-based methodology for fall detection," IEEE Sensors Journal, 2017.

[13] Panagiotis Tsinganos, and Athanassios Skodras. "A Smartphone-based Fall Detection System for the Elderly," 10th International Symposium on Image and Signal Processing and Analysis, 2012. 